\title{
COLORINGS OF TREES WITH LINEAR, INTERMEDIATE AND EXPONENTIAL SUBBALL COMPLEXITY
}

\author{
Seul Bee Lee And Seonhee Lim
}

\begin{abstract}
We study colorings of regular trees using subball complexity $b(n)$, which is the number of colored $n$-balls up to color-preserving isomorphisms.

We show that for any $k$-regular tree, for $k>1$, there are colorings of intermediate complexity. We then construct colorings of linear complexity $b(n)=2 n+2$. We also construct colorings induced from sequences of linear subword complexity which has exponential subball complexity.
\end{abstract}

\section{Introduction}

Consider a coloring $\phi: V T \rightarrow \mathcal{A}$ of a locally finite tree $T$, i.e., a coloring of vertices $V T$ of $T$, with colors in a finite set $\mathcal{A}$ (called an alphabet). The subball complexity $b_{\phi}(n)$ of a coloring $\phi$, defined by D. Kim and the second author (which is called subword complexity of a coloring in [5]), is the number of colored $n$-balls in the colored tree $(T, \phi)$ up to color-preserving isomorphisms (see the paragraph after Definition 2.2). The subball complexity $b_{\phi}(n)$ is closely related to the well-known subword complexity of a sequence, which is an important tool to study sequences which are not periodic.

The subword complexity $p_{\mathbf{w}}(n)$ of a sequence $\mathbf{w}$ is the number of distinct subwords of length $n$. Coven and Hedlund showed that the subword complexity of a two-sided sequence is bounded if and only if it is periodic [3]. A non-eventually periodic sequence with minimal unbounded subword complexity $p(n)=n+1$ is called a Sturmian sequence. It is known that Sturmian sequences are completely characterized as irrational rotations on the unit circle (see for example [7]).

D. Kim and the second author showed a theorem analogous to CovenHedlund Theorem for colorings of regular trees, namely that $b_{\phi}(n)$ is bounded

Received June 27, 2014; Revised January 13, 2015.

2010 Mathematics Subject Classification. Primary 37B10, 37E25, 05C05.

Key words and phrases. trees, colorings of trees, subword complexity, symbolic dynamics, Sturmian sequences, Sturmian colorings.

We would like to thank Donghan Kim for helpful comments. This work was supported by NRF-2013R1A1A2011942 and NRF-20100019516. The second author was supported by Korea Institute for Advanced Study (KIAS). 
if and only if $\phi$ is periodic. They further studied Sturmian colorings, which are colorings of minimal unbounded subball complexity $b(n)=n+2$ (see [5]).

In this paper, we continue the study of subball complexity of colorings of trees. For any given sequence, we first construct a coloring of subball complexity which is asymptotically of the same type as the subword complexity of the sequence.

More precisely, let $\phi_{0}$ be a two-sided sequence. We can consider $\phi_{0}$ as a coloring on vertices of a connected graph $X$, which is a 2-regular tree with one loop attached to each vertice. Given an index map $i$ on $X$, let us denote by $\pi$ the projection map from the universal covering tree $T$ of $(X, i)$ to $X$. The lift $\phi=\phi_{0} \circ \pi$ is a coloring of $T$, which we call the lifting of $\phi_{0}$ to $T$. By constructing an index map $i$ on $X$ which preserves the subball complexity, we show the following theorem (see Theorem 3.6).

Theorem 1. Let $\phi_{0}$ be a two-sided sequence with a finite alphabet $\mathcal{A}$. For any integer $k>2$, there exists a coloring $\phi: V T \rightarrow \mathcal{A}$ on a $k$-regular tree $T$ such that

$$
b_{\phi}(n)=b_{\phi_{0}}(n) .
$$

A non-decreasing function is called intermediate if it grows faster than any polynomial function and slower than any exponential function. One-sided sequences of intermediate subword complexity have been constructed by many authors (see for example [2] and [6]). For a given one-sided sequence, it is straightforward to construct a two-sided sequence whose subball complexity is of the same asymptotic type as the subword complexity of the given one-sided sequence.

Corollary 1. There exist colorings of intermediate subball complexity.

In the second part, we construct colorings with the exact subball complexity (see Theorem 4.9).

Theorem 2. For any $k>1$, there exists a family of colorings of $k$-regular trees with subball complexity $b(n)=2 n+2$.

G. Rote constructed one-sided sequences with subword complexity $p(n)=2 n$ using rotations on the unit circle (see [8] for details and examples). He showed that a sequence $\mathbf{v}=\left(v_{i}\right)_{i \in \mathbb{N}}$ is complementation-symmetric (see Definition 4.2) and $p_{\mathbf{v}}(n)=2 n$ if and only if its difference sequence $\mathbf{d}=\left(d_{i}\right)_{i \in \mathbb{N}}$ defined by $d_{i}=v_{i+1}-v_{i}(\bmod 2)$ is Sturmian.

In Section 4, we give a method of constructing non-eventually periodic twosided sequences with subword complexity $2 n$ using the method of G. Rote (see Proposition 4.6). Then, we show that if $\mathbf{w}$ is a non-eventually periodic complementation-symmetric two-sided sequence with subword complexity $p(n)$ $=2 n$, then $\mathbf{w}$ is a coloring of a 2-regular tree with subball complexity $b(n)=$ $2 n+2$ (see Theorem 4.9). 
One might ask if the asymptotic growth type of subball complexity function is preserved by any index map $i$. In Section 5, we will show that there is an index map $i$ on a 2-regular tree such that the subball complexity $b_{\phi}(n)$ is exponential (see Theorem 5.1).

Theorem 3. Let $X$ be a 2-regular tree with loops attached and $\phi_{0}$ be a coloring of $X$ on a finite alphabet $\mathcal{A}$ with unbounded subball complexity $b_{\phi_{0}}(n)$. If the 1-balls colored by $[a a a]$ occur only finitely many times in $X$ for any a $\in \mathcal{A}$, then there is an index map $i$ on $X$ such that $b_{\phi}(n)$ is an exponential function where $\phi$ is the lifting of $\phi_{0}$ to the universal covering of $(X, i)$. In particular, there are some Sturmian sequences whose induced coloring of an index map has exponential subball complexity.

\section{Preliminaries}

\subsection{Words and sequences}

An element of an alphabet $\mathcal{A}$ is called a letter. For a word of length $n \in \mathbb{N}$ (or an $n$-word) $\mathbf{u}=u_{1} u_{2} \cdots u_{n}, u_{i} \in \mathcal{A}$, the reversed word of $\mathbf{u}$ is $\overline{\mathbf{u}}=u_{n} \cdots u_{2} u_{1}$. The word $\mathbf{u}$ is called a palindrome if $\overline{\mathbf{u}}=\mathbf{u}$.

Let $\mathbf{u}$ be a sequence, by which we mean either a finite word or an infinite sequence. If there are suitable sequences $\mathbf{x}, \mathbf{y}$ and $\mathbf{z}$ such that $\mathbf{u}=\mathbf{x y z}$, then we say that $\mathbf{y}$ is admissible in $\mathbf{u}$ and that $\mathbf{y}$ is a subword of $\mathbf{u}$. The sequence $\mathbf{x}$ is called a prefix of $\mathbf{u}$ and $\mathbf{z}$ is called a suffix of $\mathbf{u}$. Let $F_{n}(\mathbf{u})$ be the set of $n$-subwords of $\mathbf{u}$ and $F(\mathbf{u})$ be the set of all subwords of $\mathbf{u}$. We say that $\mathbf{u}$ is reversible if $\overline{\mathbf{y}} \in F(\mathbf{u})$ for any $\mathbf{y} \in F(\mathbf{u})$.

The subword complexity $p_{\mathbf{u}}(n)$ of a sequence $\mathbf{u}$ is the number of distinct $n$-subwords:

$$
p_{\mathbf{u}}(n)=\left|F_{n}(\mathbf{u})\right| \text {. }
$$

A sequence $\mathbf{u}=\left(u_{i}\right)$ is periodic if there is $p \in \mathbb{N}$ such that $u_{i}=u_{i+p}$ for any $i$. A one-sided sequence $\mathbf{v}=v_{1} v_{2} \ldots$ is eventually periodic if $v_{k} v_{k+1} \ldots$ is periodic for some $k \in \mathbb{N}$. A two-sided sequence $\mathbf{w}=\cdots w_{-1} w_{0} w_{1} \cdots$ is eventually periodic if both $\cdots w_{-k-1} w_{-k}$ and $w_{k} w_{k+1} \cdots$ are periodic for some $k \in \mathbb{N}$.

Coven and Hedlund showed that subword complexity of a one-sided (twosided, respectively) sequence is bounded if and only if the one-sided (two-sided, respectively) sequence is eventually periodic (periodic, respectively).

A one-sided sequence $\mathbf{v}$ with minimal unbounded subword complexity $p_{\mathbf{v}}(n)$ $=n+1$ is called a one-sided Sturmian sequence. A non-eventually periodic twosided sequence $\mathbf{w}$ with $p_{\mathbf{w}}(n)=n+1$ is called a two-sided Sturmian sequence. It is known that a Sturmian sequence is induced by an irrational circle rotation as follows.

Proposition 2.1 ([7], p. 51, Theorem 2.1.13). A one-sided (or two-sided, respectively) sequence $\mathbf{u}$ is Sturmian if and only if there exist $c \in \mathbb{R}$ and $\theta \in \mathbb{R} \backslash \mathbb{Q}$ 
where $0<\theta<1$ such that $\mathbf{u}=\left(u_{m}\right)_{m \in \mathbb{N}}\left(\right.$ or $\mathbf{u}=\left(u_{m}\right)_{m \in \mathbb{Z}}$, respectively) is defined by

or

$$
u_{m}= \begin{cases}1, & \text { if } c+m \theta(\bmod 1) \in[0, \theta) \\ 0, & \text { if } c+m \theta(\bmod 1) \in[\theta, 1)\end{cases}
$$

$$
u_{m}= \begin{cases}1, & \text { if } c-m \theta(\bmod 1) \in[0, \theta) \\ 0, & \text { if } c-m \theta(\bmod 1) \in[\theta, 1) .\end{cases}
$$

\subsection{Colorings of trees}

A tree $T$ is a connected graph without cycles. Let $V T$ be the vertex set of $T$ and $E T$ be the edge set of $T$ which is the set of oriented edges of $T$. We endow $T$ with the metric $d$ for which each edge has length 1 . Denote by $\bar{e}$ the inverse of an edge $e$, i.e., the same edge as $e$ with inverse orientation. An edge which is directed from $v$ to $w$ for $v, w \in V T$ will be denoted by $[v, w]$.

Let $\partial_{0}(e)$ be the initial vertex of $e$ and $\partial_{1}(e)$ be the terminal vertex of $e$. Consider $\partial_{0}$ and $\partial_{1}$ as maps from $E T$ to $V T$. For a fixed $k \in \mathbb{N}$, a tree $T$ is called $k$-regular if $\left|\partial_{0}^{-1}(v)\right|=k$ for every $v \in V T$.

An $n$-ball around $x \in V T$ is defined by $B_{n}(x)=\{y \in T: d(x, y) \leq n\}$. An $n$-sphere around $x$ is defined by $S_{n}(x)=\{y \in T: d(x, y)=n\}$.

Definition 2.2. Let $T$ be a $k$-regular tree. A coloring of a tree is a map $\phi: V T \rightarrow \mathcal{A}$ where $\mathcal{A}$ is an alphabet.

Let $T$ be a $k$-regular tree and $\phi$ be a coloring of $T$. Let $T_{1}$ and $T_{2}$ be subtrees of $T$. A color-preserving map $f: T_{1} \rightarrow T_{2}$ is a graph homomorphism such that $\phi(v)=\phi(f(v))$ for $v \in V T_{1}$. We say that $B_{n}(x)$ and $B_{n}(y)$ are equivalent if there exists a color-preserving isomorphism $f: B_{n}(x) \rightarrow B_{n}(y)$. We call such an equivalence class a colored $n$-ball and denote it by $\left[B_{n}(x)\right]$. The set of colored $n$-balls is denoted by $\mathcal{B}_{\phi}(n)$.

Definition 2.3 ([5]). The subball complexity of a coloring $\phi$ is a fuction $b_{\phi}$ : $\mathbb{Z}_{\geq 0} \rightarrow \mathbb{N}$ defined by $b_{\phi}(n)=\left|\mathcal{B}_{\phi}(n)\right|$.

\subsection{Graph of groups and edge-indexed graphs}

Definition $2.4([9])$. A graph of groups $\mathcal{G}=\left(X, A_{\bullet}\right)$ consists of a connected graph $X$, a collection of groups $\left\{A_{x}\right\}_{x \in V X \sqcup E X}$ such that $A_{e}=A_{\bar{e}}$ for each $e \in E X$ and a collection of monomorphisms $\left\{\alpha_{e}: A_{e} \rightarrow A_{\partial_{0}(e)}\right\}_{e \in E X}$.

Definition 2.5 ([5]). An edge-indexed graph is a connected graph $X$ with an index map $i: E X \rightarrow \mathbb{N}$ assigning a cardinal number $i(e)$ to every oriented edge $e$.

The universal covering of an edge-indexed graph $(X, i)$ is constructed as follows. Choose a vertex $v_{0}$ of $X$ and an edge $e$ with $\partial_{0}(e)=v_{0}$. Start with a vertex, say $\widetilde{v_{0}}$. Attach $i(e)$ edges $\widetilde{e}_{j}, j=1, \ldots, i(e)$ to $\widetilde{v_{0}}$ as liftings of $e$. Let $e^{\prime}$ be an edge of $X$ such that $\partial_{1}(e)=\partial_{0}\left(e^{\prime}\right)$. For each edge $\widetilde{e}_{j}$, attach $i\left(e^{\prime}\right)$ edges $\widetilde{e_{k}^{\prime}}, k=1, \ldots, i\left(e^{\prime}\right)$ to $\partial_{1}\left(\widetilde{e}_{j}\right)$ as liftings of $e^{\prime}$. We obtain the universal covering 
tree $T$ of $(X, i)$ by repeating this procedure. Remark that graphs of groups are always developable as opposed to complexes of groups in dimension at least 2 .

Remark. Let $T$ be a $k$-regular tree colored by $\phi$ and $G$ be the set of automorphisms of $T$. Let $\Gamma_{\phi}$ be the group of color-preserving automorphisms. We call $\gamma \in G$ an inversion if $\gamma(e)=\bar{e}$ for an edge $e$.

If $\Gamma_{\phi}$ does not contain inversions, then $\Gamma_{\phi} \backslash T$ is a graph without any loop, i.e., without any edge $e$ such that $\partial_{0}(e)=\partial_{1}(e)$. If $\Gamma_{\phi}$ contains inversions, then $\Gamma_{\phi} \backslash T$ has a loop. Then, we can take a barycentric subdivision $T^{\prime}$ on the edges of $T$ stabilized by inversions in $\Gamma_{\phi}$. The vertices of $T$ will be expressed as black vertices and the vertices of $T^{\prime}-T$ will be expressed by white vertices.

Let $\pi: T^{\prime} \rightarrow X=\Gamma_{\phi} \backslash T^{\prime}$ be the covering map. Let us denote by $\widetilde{x} \in$ $V T^{\prime} \sqcup E T^{\prime}$ a lifting of $x \in V X \sqcup E X$. In $\Gamma_{\phi}$, the group of stabilizers of $\widetilde{x}$ is denoted by $\Gamma_{\widetilde{x}}$. If $\pi(\widetilde{x})=\pi(\widetilde{y})$, then $\Gamma_{\widetilde{x}}$ and $\Gamma_{\widetilde{y}}$ are isomorphic.

We define the quotient graph of groups $\Gamma_{\phi} \backslash \backslash T^{\prime}=\left(X, A_{\bullet}\right)$ as follows. For $x \in V X \sqcup E X$, we define $A_{x}$ as $\Gamma_{\widetilde{x}}$. For $e \in E X, \gamma\left(\partial_{0}(\widetilde{e})\right)=\widetilde{\partial_{0}(e)}$ for some $\gamma \in \Gamma_{\phi}$. Let $f_{\gamma}: \Gamma_{\partial_{0}(\widetilde{e})} \rightarrow \Gamma_{\widetilde{\partial_{0}(e)}}$ be an isomorphism defined by $f_{\gamma}(g)=\gamma g \gamma^{-1}$ and $\iota_{\widetilde{e}}$ be the inclusion from $\Gamma_{\widetilde{e}}$ to $\Gamma_{\partial_{0}(\widetilde{e})}$. A monomorphism $\alpha_{e}: A_{e} \rightarrow A_{\partial_{0}(e)}(\cong$ $\left.\Gamma_{\widetilde{e}} \rightarrow \Gamma_{\widetilde{\partial_{0}(e)}}\right)$ is defined by $\alpha_{e}=f_{\gamma} \circ \iota_{\widetilde{e}}$. Hence, $\Gamma_{\phi} \backslash \backslash T^{\prime}=\left(X, A_{\bullet}\right)$ is a graph of groups. The universal covering of $\Gamma_{\phi} \backslash \backslash T^{\prime}$ is $T^{\prime}$ [1, p. 20].

We define an index map $i: E X \rightarrow \mathbb{N}$ by $e \mapsto\left[A_{\partial_{0}(e)}: \alpha_{e} A_{e}\right]$. The universal covering of $(X, i)$ is isomorphic to the universal covering of $\Gamma_{\phi} \backslash \backslash T^{\prime}$, thus isomorphic to $T^{\prime}$.

\section{From one-sided sequences to colorings}

For a given one-sided sequence $\mathbf{v}=v_{1} v_{2} \cdots$, we can find a two-sided sequence with the same asymptotic growth type of subword complexity, simply by taking $\mathbf{w}=\cdots \cdots v_{3} v_{2} v_{1} v_{2} v_{3} \cdots$. It is easy to see that

$$
p_{\mathbf{v}}(n) \leq p_{\mathbf{w}}(n) \leq 2 p_{\mathbf{v}}(n)+n-2 .
$$

Considering $\mathbf{w}$ as a coloring, a subword, as a sub-ball, is equivalent to its reversed word. Thus

$$
\frac{p_{\mathbf{v}}(2 n+1)}{2} \leq b_{\mathbf{w}}(n) \leq p_{\mathbf{v}}(2 n+1)+n .
$$

Here, $b_{\mathbf{w}}(n)$ is the subball complexity of $\mathbf{w}$ seen as a coloring on a 2-regular tree. It follows that if there is a one-sided sequence $\mathbf{v}$ of polynomial subword complexity $p_{\mathbf{v}}(n)$, then one can construct a coloring $\mathbf{w}$ of 2-regular tree with subball complexity $b_{\mathbf{w}}(n)$ with the same asymptotic type as $p_{\mathbf{v}}(n)$, i.e.,

$$
b_{\mathbf{w}}(n)=O\left(p_{\mathbf{v}}(n)\right) \text { and } p_{\mathbf{v}}(n)=O\left(b_{\mathbf{w}}(n)\right) .
$$

In this section, we extend the above idea to construct colorings of $k$-regular trees induced from a one-sided sequence $\mathbf{v}$, for any $k$, with subball complexity 
$b(n)$ with the same asymptotic type as $p_{\mathbf{v}}(n)$. We further construct some sequences whose subball complexity is precisely $2 n+2$.

\subsection{From one-sided sequences to two-sided sequences}

For some special one-sided sequences, there exist two-sided sequences with the same subword complexity.

Proposition 3.1. Let $\mathbf{u}=u_{1} u_{2} \cdots$ be a one-sided sequence. If every prefix of $\mathbf{u}$ of length $n$ is a suffix of an $(n+1)$-subword of $\mathbf{u}$, then there is a two-sided sequence $\mathbf{w}$ such that $F(\mathbf{u})=F(\mathbf{w})$. It follows that

$$
p_{\mathbf{w}}(n)=p_{\mathbf{u}}(n) .
$$

Proof. We first claim that there is a letter $a \in \mathcal{A}$ such that $F(a \mathbf{u})=F(\mathbf{u})$, and that every prefix of $a \mathbf{u}$ of length $n$ is a suffix of an $(n+1)$-subword of $a \mathbf{u}$.

Suppose that for each letter $a$, there is a positive integer $N_{a}$ such that $a u_{1} u_{2} \cdots u_{N_{a}}$ is not admissible in $\mathbf{u}$. Let $N=\max \left\{N_{a}: a \in \mathcal{A}\right\}$. On the other hand, by the assumption, there is a letter $b$ such that $b u_{1} u_{2} \cdots u_{N}$ is admissible in $\mathbf{u}$. Since $N \geq N_{b}, b u_{1} u_{2} \cdots u_{N_{b}}$ is also admissible in $\mathbf{u}$, which is a contradiction.

Therefore, there is a letter $a$ such that $a u_{1} u_{2} \cdots u_{n} \in F(\mathbf{u})$ for all $n$. Thus $F(a \mathbf{u})=F(\mathbf{u})$. Therefore, a prefix of $a \mathbf{u}$ of length $n$ is a subword of $\mathbf{u}$, thus it is a suffix of an $(n+1)$ - subword of $a \mathbf{u}$.

Since $a \mathbf{u}$ satisfies the assumption again, there is a letter $b$ such that $F(b a \mathbf{u})=$ $F(\mathbf{u})$. We obtain a two-sided sequence $\mathbf{w}$ such that $F(\mathbf{w})=F(\mathbf{u})$ by repeating this process.

Example 3.2. Consider sequences satisfying Proposition 3.1:

$$
010110101100101 \cdots \text { and } 001001111001001 \cdots \text { for example. }
$$

Subwords of those sequences occur infinitely many times. Thus they satisfy the assumption of Proposition 3.1 (see Section 3 of [8] for details).

\subsection{From colorings of a 2-regular tree to colorings of a $k$-regular tree}

In this subsection, we show that for a given coloring $\phi_{0}$ of 2-regular tree, there exists a coloring $\phi$ with the same subball complexity.

We will use notations introduced in Section 2.3. Let us fix a coloring $\phi_{0}$ on a tree $T$. Throughout this subsection, let $x_{i}, y_{i}, i=1, \ldots, k$ be the neighboring vertices of $x, y$, respectively.

Definition 3.3. The $n$-branch $B r_{n}\left(x, x_{i}\right)$ from $x$ to $x_{i}$ is defined as the set $\left[x, x_{i}\right] \cup\left\{y \in B_{n}(x) \mid d\left(y, x_{i}\right)<d(y, x)\right\}$. Let us denote by $\left[B r_{n}\left(x, x^{\prime}\right)\right]$ the equivalence class of colored branch $B r_{n}\left(x, x^{\prime}\right)$ up to color-preserving isomorphisms. We will say that $\left[B r_{n}\left(x, x^{\prime}\right)\right]=\left[B r_{n}\left(y, y^{\prime}\right)\right]$ via $f$ if there exists a color-preserving isomorphism $f: B r_{n}\left(x, x^{\prime}\right) \rightarrow B r_{n}\left(y, y^{\prime}\right)$. 


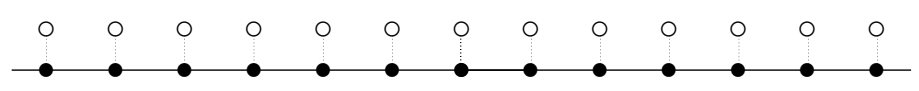

FIGURE 1

Lemma 3.4 ([5], Lemma 2). We have $\left[B_{n}(x)\right]=\left[B_{n}(y)\right]$ if and only if there exists a permutation $\sigma \in S_{k}$ such that for all $i=1, \ldots, k$, we have $\left[B r_{n}\left(x, x_{i}\right)\right]=$ $\left[B r_{n}\left(y, y_{\sigma(i)}\right)\right]$. Consequently, if $\left[B r_{n}\left(x, x^{\prime}\right)\right]=\left[B r_{n}\left(y, y^{\prime}\right)\right]$ and $\left[B r_{n+1}\left(x^{\prime}, x\right)\right]$ $=\left[B r_{n+1}\left(y^{\prime}, y\right)\right]$, then $\left[B_{n}(x)\right]=\left[B_{n}(y)\right]$.

Throughout this subsection, let $X$ be a graph which is a 2-regular tree with loops possibly attached as in Figure 1 (see the remark after Definition 2.5). Let us denote $V X$ by $\left\{v_{n}\right\}_{v \in \mathbb{Z}}$ and $E X=\left\{\left[v_{j}, v_{j \pm 1}\right],\left[v_{j}, v_{j}\right]: j \in \mathbb{Z}\right\}$. The dotted lines of Figure 1 indicate that loops may or may not exist. We can define an index map $i$ on $X$ such that indices on edges starting from the white vertices are all 2 . Then, the universal covering $T^{\prime}$ of $(X, i)$ is regarded as a barycentric subdivision of $T$ as explained in Section 2.3. By abuse of notation, we will denote the universal covering of $(X, i)$ by $T$.

For $x \in V X$, let us denote by $\widetilde{x}$ its lift in $T$. We can easily check the next lemma.

Lemma 3.5. Fix $\widetilde{x}$ and let $\widetilde{x}_{i}, i=1, \ldots, k$ be the lifts of $x_{i}$ which are the neighboring vertices of $\widetilde{x} \in T$.

(1) The colors of the vertices of $B_{n}(x)$ and the indices of the edges of $B_{n}(x)-S_{n}(x)$ determine $\left[B_{n}(\widetilde{x})\right]$.

(2) The colors of the vertices of $B_{n}\left(\pi\left(\widetilde{x}_{i}\right)\right)$ and the indices of the edges of $B_{n}\left(\pi\left(\widetilde{x}_{i}\right)\right)-S_{n}\left(\pi\left(\widetilde{x}_{i}\right)\right)$ determine $\left[B r_{n+1}\left(\widetilde{x}, \widetilde{x}_{i}\right)\right]$.

Remark. Note that although there are many liftings of $x$ and $x_{i}$, the class $\left[B r_{n}\left(\widetilde{x}, \widetilde{x}_{i}\right)\right]$ is unique. Also, if $\pi\left(\widetilde{x}_{i}\right)=\pi\left(\widetilde{x}_{j}\right)$, then $\left[B r_{n}\left(\widetilde{x}, \widetilde{x}_{i}\right)\right]=\left[B r_{n}\left(\widetilde{x}, \widetilde{x}_{j}\right)\right]$. By abuse of terminology, let us denote by $\left[B r_{n}\left(\widetilde{x}, \widetilde{\pi\left(\widetilde{x_{i}}\right)}\right]=\left[B r_{n}\left(\widetilde{x}, \widetilde{x}_{i}\right)\right]\right.$. This notation allows us to write $\left[B r_{n}\left(\widetilde{v_{s}}, \widetilde{v_{s}}\right)\right]$, where the second $\widetilde{v_{s}}$ means actually a neighboring vertex of $\widetilde{v_{s}}$ whose projected image is $v_{s}$. This case happens when there is a loop attached to $v_{s}$.

Now we are ready to prove Theorem 1 in the introduction.

Theorem 3.6. Let $\phi_{0}$ be a coloring of a 2-regular tree $X$ with loops possibly attached. For any integer $k>2$, there exists an index map $i$ on $X$ such that

(1) the universal covering $T$ of $(X, i)$ is a k-regular tree and

(2) for any $n, b_{\phi}(n)=b_{\phi_{0}}(n)$ where $\phi$ is the lifting of $\phi_{0}$ to $T$.

Proof. Fix $k>2$. Define an index map $i: E X \rightarrow \mathbb{N}$ which satisfies the following conditions.

(i) For every $v_{t} \in V X, i\left[v_{t}, v_{t-1}\right]+i\left[v_{t}, v_{t}\right]+i\left[v_{t}, v_{t+1}\right]=k$. 
(ii) If $\left[B_{1}\left(v_{t}\right)\right]=\left[B_{1}\left(v_{s}\right)\right]$ via $f$, then $i\left[v_{t}, v_{t^{\prime}}\right]=i\left[v_{s}, f\left(v_{t^{\prime}}\right)\right], v_{t^{\prime}} \in B_{1}\left(v_{t}\right)$.

(iii) Let $\left[B_{1}\left(v_{s}\right)\right]=[a a b]$ and $\left[B_{1}\left(v_{t}\right)\right]=[b a b]$ for distinct letters $a$ and $b$. Then, $i\left[v_{s}, v_{s-l}\right]+i\left[v_{s}, v_{s}\right] \neq i\left[v_{t}, v_{t}\right]$ where $l=1$ or $l=-1$ such that $\phi_{0}\left(v_{s+l}\right)=b$.

Condition (i) ensures that the universal covering $T$ of $(X, i)$ is a $k$-regular tree. Condition (ii) ensures that if $\left[B_{1}\left(v_{t}\right)\right]=\left[B_{1}\left(v_{s}\right)\right]$, then $\left[B_{1}\left(\widetilde{v}_{t}\right)\right]=\left[B_{1}\left(\widetilde{v}_{s}\right)\right]$. Condition (iii) ensures that if $\left[B_{1}\left(\widetilde{v}_{t}\right)\right]=\left[B_{1}\left(\widetilde{v_{s}}\right)\right]$, then $\left[B_{1}\left(v_{t}\right)\right]=\left[B_{1}\left(v_{s}\right)\right]$.

Since we define $i$ around each vertex independently, there is no problem of compatibility.

It is enough to show that for all $n \in \mathbb{N}$ and $v_{t}, v_{s} \in V X$,

$$
\left[B_{n}\left(v_{t}\right)\right]=\left[B_{n}\left(v_{s}\right)\right] \text { if and only if }\left[B_{n}\left(\widetilde{v_{t}}\right)\right]=\left[B_{n}\left(\widetilde{v_{s}}\right)\right]
$$

Let us prove by induction on $n$. The case when $n=1$ is clearly satisfied by conditions (ii) and (iii). Assume that (3.1) is true up to $n$ and let us prove the case $n+1$.

(a) $(\Rightarrow)$ Assume that $\left[B_{n+1}\left(v_{t}\right)\right]=\left[B_{n+1}\left(v_{s}\right)\right]$ via $f$ for some $v_{t}, v_{s} \in V X$. By the condition (ii), for all $x \in V B_{n}\left(v_{s}\right), i[x, y]=i[f(x), f(y)]$ where $y \in$ $V B_{1}(x)$. By Lemma 3.5(1), $\left[B_{n+1}\left(\widetilde{v_{t}}\right)\right]=\left[B_{n+1}\left(\widetilde{v_{s}}\right)\right]$.

(b) $(\Leftarrow)$ Let us assume that $\left[B_{m}\left(\widetilde{v_{t}}\right)\right]=\left[B_{m}\left(\widetilde{v_{s}}\right)\right]$ implies $\left[B_{m}\left(v_{t}\right)\right]=\left[B_{m}\left(v_{s}\right)\right]$ for any $v_{t}, v_{s} \in V X$ and $m=1, \ldots, n$.

Suppose that $\left[B_{n+1}\left(\widetilde{v_{s}}\right)\right]=\left[B_{n+1}\left(\widetilde{v_{t}}\right)\right]$. Then, $\left[B_{n}\left(\widetilde{v_{s}}\right)\right]=\left[B_{n}\left(\widetilde{v_{t}}\right)\right]$, thus $\left[B_{n}\left(v_{s}\right)\right]=\left[B_{n}\left(v_{t}\right)\right]$. We may assume that $\phi_{0}\left(v_{s+l}\right)=\phi_{0}\left(v_{t+l}\right)$ for all $-n \leq l \leq$ $n$. (The case $\phi_{0}\left(v_{s+l}\right)=\phi_{0}\left(v_{t-l}\right)$ can be treated similarly.) By Lemma 3.5(2),

$$
\left[B r_{n}\left(\widetilde{v_{s-1}}, \widetilde{v_{s}}\right)\right]=\left[B r_{n}\left(\widetilde{v_{t-1}}, \widetilde{v_{t}}\right)\right] \text { and }\left[B r_{n}\left(\widetilde{v_{s+1}}, \widetilde{v_{s}}\right)\right]=\left[B r_{n}\left(\widetilde{v_{t+1}}, \widetilde{v_{t}}\right)\right] \text {. }
$$

We have $\left[B r_{n+1}\left(\widetilde{v_{s}}, \widetilde{v_{s}}\right)\right]=\left[B r_{n+1}\left(\widetilde{v_{t}}, \widetilde{v_{t}}\right)\right]$ by Lemme $3.5(2)$. By the condition (ii), $i\left[v_{s}, v_{s}\right]=i\left[v_{t}, v_{t}\right]$.

By Lemma 3.4, branches going out of $\widetilde{v_{s}}$ correspond to those out of $\widetilde{v_{t}}$, thus it is sufficient to consider the next two cases.

(1) If $\left[B r_{n+1}\left(\widetilde{v_{s}}, \widetilde{v_{s-1}}\right)\right]=\left[B r_{n+1}\left(\widetilde{v_{t}}, \widetilde{v_{t-1}}\right)\right]$ and $\left[B r_{n+1}\left(\widetilde{v_{s}}, \widetilde{v_{s+1}}\right)\right]=$ $\left[B r_{n+1}\left(\widetilde{v_{t}}, \widetilde{v_{t+1}}\right)\right]$, by Lemma 3.4 and $(3.2),\left[B_{n}\left(\widetilde{v_{s-1}}\right)\right]=\left[B_{n}\left(\widetilde{v_{t-1}}\right)\right]$ and $\left[B_{n}\left(\widetilde{v_{s+1}}\right)\right]=\left[B_{n}\left(\widetilde{v_{t+1}}\right)\right]$. By induction hypothesis, $\left[B_{n}\left(v_{s-1}\right)\right]=\left[B_{n}\left(v_{t-1}\right)\right]$ and $\left[B_{n}\left(v_{s+1}\right)\right]=\left[B_{n}\left(v_{t+1}\right)\right]$. Thus, $\left[B_{n+1}\left(v_{s}\right)\right]=\left[B_{n+1}\left(v_{t}\right)\right]$.

(2) If $\left[B r_{n+1}\left(\widetilde{v_{s}}, \widetilde{v_{s-1}}\right)\right]=\left[B r_{n+1}\left(\widetilde{v_{t}}, \widetilde{v_{t+1}}\right)\right]$ and $\left[B r_{n+1}\left(\widetilde{v_{s}}, \widetilde{v_{s+1}}\right)\right]=$ $\left[B r_{n+1}\left(\widetilde{v_{t}}, \widetilde{v_{t-1}}\right)\right]$, we obtain the next claim.

Claim: Let $1 \leq m \leq n$. If $\phi_{0}\left(v_{s+l}\right)=\phi_{0}\left(v_{t-l}\right)$ for all $-m \leq l \leq m$, then $\phi_{0}\left(v_{s+l}\right)=\phi_{0}\left(v_{t-l}\right)$ for all $-(m+1) \leq l \leq(m+1)$.

Proof of Claim. Suppose that $\phi_{0}\left(v_{s+l}\right)=\phi_{0}\left(v_{t-l}\right)$ for all $-m \leq l \leq m$. Then, $\left[B r_{m}\left(\widetilde{v_{s-1}}, \widetilde{v_{s}}\right)\right]=\left[B r_{m}\left(\widetilde{v_{t+1}}, \widetilde{v_{t}}\right)\right]$ and $\left[B r_{m}\left(\widetilde{v_{s+1}}, \widetilde{v_{s}}\right)\right]=\left[B r_{m}\left(\widetilde{v_{t-1}}, \widetilde{v_{t}}\right)\right]$ by Lemma 3.5(2).

Since $\left[B r_{m+1}\left(\widetilde{v_{s}}, \widetilde{v_{s-1}}\right)\right]=\left[B r_{m+1}\left(\widetilde{v_{t}}, \widetilde{v_{t+1}}\right)\right]$ and $\left[B r_{m+1}\left(\widetilde{v_{s}}, \widetilde{v_{s+1}}\right)\right]=$ $\left[B r_{m+1}\left(\widetilde{v_{t}}, \widetilde{v_{t-1}}\right)\right],\left[B_{m}\left(\widetilde{v_{s-1}}\right)\right]=\left[B_{m}\left(\widetilde{v_{t+1}}\right)\right]$ and $\left[B_{m}\left(\widetilde{v_{s+1}}\right)\right]=\left[B_{m}\left(\widetilde{v_{t-1}}\right)\right]$ (see Lemma 3.4). By induction hypothesis, $\left[B_{m}\left(v_{s-1}\right)\right]=\left[B_{m}\left(v_{t+1}\right)\right]$ and 
$\left[B_{m}\left(v_{s+1}\right)\right]=\left[B_{m}\left(v_{t-1}\right)\right]$. By the assumption of Claim, $\phi_{0}\left(v_{s-m-1}\right)=$ $\phi_{0}\left(v_{t+m+1}\right)$ and $\phi_{0}\left(v_{s+m+1}\right)=\phi_{0}\left(v_{t-m-1}\right)$.

Now since $\phi_{0}\left(v_{s-1}\right)=\phi_{0}\left(v_{t+1}\right)$ and $\phi_{0}\left(v_{s+1}\right)=\phi_{0}\left(v_{t-1}\right), \phi_{0}\left(v_{s+l}\right)=$ $\phi_{0}\left(v_{t-l}\right)$ for all $-(n+1) \leq l \leq(n+1)$, i.e., $\left[B_{n+1}\left(v_{s}\right)\right]=\left[B_{n+1}\left(v_{t}\right)\right]$.

By induction, (3.1) holds for any $n$.

Corollary 3.7. For any $k \in \mathbb{N}_{\geq 2}$, there exists a coloring of a $k$-regular tree of intermediate subball complexity.

Proof. Recall that there are sequences of intermediate subword complexity $p_{\mathbf{u}}(n)$ [2], thus by the remark in the beginning of this section, there exists a coloring of a 2-regular tree with intermediate subball complexity $b_{\mathbf{w}}(n)$. By the above theorem, there exists a coloring of a $k$-regular tree of intermediate subball complexity $b_{\phi}(n)$.

\section{Linear subball complexity}

\subsection{Colorings with subball complexity $2 n+2$}

In this section, we construct colorings with subball complexity $2 n+2$ using a family of one-sided sequences with $p(n)=2 n$ constructed by G. Rote.

Proposition 4.1 ([8], Theorem 2). Let c, $\varphi \in \mathbb{R}$ and $\theta \in \mathbb{R} \backslash \mathbb{Q}$ such that $0<\varphi<1,0<\theta<\min \{\varphi, 1-\varphi\}$ and $m \theta \not \equiv \varphi(\bmod 1)$ for any $m \in \mathbb{Z}$. Then, a one-sided sequence $\mathbf{v}=v_{1} v_{2} \cdots v_{m} \cdots$ defined by

$$
v_{m}=\left\{\begin{array}{ll}
1, & \text { if } c+m \theta(\bmod 1) \in[0, \varphi) \\
0, & \text { if } c+m \theta(\bmod 1) \in[\varphi, 1)
\end{array} \quad \text { for each } m \in \mathbb{N}\right.
$$

has subword complexity $p_{\mathbf{v}}(n)=2 n$.

Definition 4.2. Let $\mathbf{u}$ be a sequence over $\{0,1\}$. Denote by $\widehat{\mathbf{u}}$ a sequence obtained by interchanging 0 and 1 of $\mathbf{u}$. The sequence $\mathbf{u}$ is called complementationsymmetric if $\widehat{\mathbf{y}} \in F(\mathbf{u})$ for any $\mathbf{y} \in F(\mathbf{u})$. The difference sequence $\mathbf{d}(\mathbf{u})=\left(d_{i}\right)$ of $\mathbf{u}=\left(u_{i}\right)$ is defined by $d_{i}=u_{i+1}-u_{i}(\bmod 2)$.

It follows that

$$
u_{i}=u_{1}+d_{1}+\cdots+d_{i-1}(\bmod 2) .
$$

Lemma 4.3. Let $\mathbf{x}=x_{1} x_{2} \cdots x_{n}$ and $\mathbf{y}=y_{1} y_{2} \cdots y_{n}$ be $n$-words over $\{0,1\}$. Then $\mathbf{d}(\mathbf{x})=\mathbf{d}(\mathbf{y})$ if and only if $\mathbf{x}=\mathbf{y}$ or $\widehat{\mathbf{x}}=\mathbf{y}$.

Proof. $(\Leftarrow)$ Clearly, it is sufficient to show that $\mathbf{d}(\mathbf{x})=\mathbf{d}(\widehat{\mathbf{x}})$. Note that $\widehat{\mathbf{x}}=$ $\widehat{x_{1} x_{2}} \cdots \widehat{x_{n}}$. Since $x_{i+1}-x_{i}=\widehat{x_{i+1}}-\widehat{x_{i}}(\bmod 2), \mathbf{d}(\mathbf{x})=\mathbf{d}(\widehat{\mathbf{x}})$.

$(\Rightarrow)$ Suppose that $\mathbf{d}(\mathbf{x})=\mathbf{d}(\mathbf{y})$. Since $\mathbf{d}(\mathbf{y})=\mathbf{d}(\widehat{\mathbf{y}}), \mathbf{d}(\mathbf{x})=\mathbf{d}(\widehat{\mathbf{y}})$. If $x_{1}=y_{1}$, then $\mathbf{x}=\mathbf{y}$ by (4.2). If $x_{1} \neq y_{1}$, then $x_{1}=\widehat{y_{1}}$. By $(4.2), \mathbf{x}=\widehat{\mathbf{y}}$. 
Remark that a sequence $\mathbf{u}$ over $\{0,1\}$ is periodic (eventually periodic, respectively) if and only if $\mathbf{d}(\mathbf{u})$ is periodic (eventually periodic, respectively).

Using Proposition 2.1 and Proposition 4.1, G. Rote showed the following proposition.

Proposition 4.4 ([8], Theorem 3 and Corollary). Let $\mathbf{v}$ be a one-sided sequence over $\{0,1\}$. The followings are equivalent.

(1) The sequence $\mathbf{v}$ is complementation-symmetric and $p_{\mathbf{v}}(n)=2 n$.

(2) The difference seuquence $\mathbf{d}(\mathbf{v})$ is Sturmian.

(3) The sequence $\mathbf{v}$ is constructed as in Proposition 4.1 with $\varphi=1 / 2$.

Lemma 4.5. Let $\mathbf{w}=\cdots w_{-1} w_{0} w_{1} \cdots$ be a two-sided sequence. Define $\mathbf{w}_{\geq l}$ as $w_{l} w_{l+1} \cdots$ for each $l \in \mathbb{Z}$.

(1) Then, $F(\mathbf{w})=\bigcup_{l \in \mathbb{Z}} F\left(\mathbf{w}_{\geq l}\right)$ and

(2) If $\mathbf{w}_{\geq l}$ is non-eventually periodic for some $l \in \mathbb{Z}$, then $\mathbf{w}$ is noneventually periodic.

(3) Let $f: \mathbb{N} \rightarrow \mathbb{N}$ be a function. Suppose that $p_{\mathbf{w}>l}(n)=f(n)$ for any $l \in \mathbb{Z}$. Then, $F(\mathbf{w})=F\left(\mathbf{w}_{\geq l}\right)$ for any $l \in \mathbb{Z}$, i.e., $p_{\mathbf{w}}(n)=f(n)$.

Proof. (1) It is clear that $\bigcup_{l \in \mathbb{Z}} F\left(\mathbf{w}_{\geq l}\right) \subset F(\mathbf{w})$. Consider a subword $\mathbf{u}=$ $w_{l} w_{l+1} \cdots w_{l+n}$ for some $n \in \mathbb{N}$. Since $\mathbf{u}$ is a subword of $\mathbf{w}_{\geq l}, \mathbf{u} \in \bigcup_{l \in \mathbb{Z}} F\left(\mathbf{w}_{\geq l}\right)$.

(2) By the definition, it is trivial.

(3) Let $m \in \mathbb{Z}$. Since $F\left(\mathbf{w}_{\geq l+1}\right) \subset F\left(\mathbf{w}_{\geq l}\right)$ and $p_{\mathbf{w} \geq l}(n)=p_{\mathbf{w}>l+1}(n)$, $F\left(\mathbf{w}_{\geq l+1}\right)=F\left(\mathbf{w}_{\geq l}\right)$. By part $(1), F(\mathbf{w})=F\left(\mathbf{w}_{\geq l}\right)$ for any $l$.

Proposition 4.6. Let $c, \varphi$ and $\theta$ as in Proposition 4.1. Then, a two-sided sequence $\mathbf{w}=\cdots w_{-1} w_{0} w_{1} \cdots w_{m} \cdots$ defined by

$$
w_{m}=\left\{\begin{array}{ll}
1, & \text { if } c+m \theta(\bmod 1) \in[0, \varphi) \\
0, & \text { if } c+m \theta(\bmod 1) \in[\varphi, 1)
\end{array} \quad \text { for each } m \in \mathbb{Z}\right.
$$

is a non-eventually periodic two-sided sequence with $p_{\mathbf{w}}(n)=2 n$.

Proof. Let $\mathbf{w}$ be a two-sided sequence which is defined by (4.3) with $c=c_{\mathbf{w}}$, $\varphi=\varphi_{\mathbf{w}}$ and $\theta=\theta_{\mathbf{w}}$ as in Proposition 4.1.

For any $m \in \mathbb{Z}, \mathbf{w}_{\geq l}$ is constructed as Proposition 4.1 with $c=c_{\mathbf{w}}+(l-$ 1) $\theta_{\mathbf{w}}, \varphi=\varphi_{\mathbf{w}}$ and $\theta=\theta_{\mathbf{w}}$. Thus, $p_{\mathbf{w}>l}(n)=2 n$ for any $l$. By Lemma 4.5 , $p_{\mathbf{w}}(n)=2 n$. Since $p_{\mathbf{w} \geq l}(n)=2 n, \mathbf{w}_{\geq l}$ is a non-eventually periodic one-sided sequence by Coven-Hedlund Theorem. Thus, $\mathbf{w}$ is non-eventually periodic (see Lemma 4.5(2)).

Proposition 4.7. Let $\mathbf{w}$ be a two-sided sequence over $\{0,1\}$. The following conditions are equivalent.

(i) For all $l \in \mathbb{Z}, \mathbf{w}_{\geq l}$ is constructed as in Proposition 4.1 with $\varphi=1 / 2$.

(ii) The sequence $\mathbf{w}$ is constructed as in Proposition 4.6 with $\varphi=1 / 2$.

(iii) For all $l \in \mathbb{Z}, \mathbf{d}\left(\mathbf{w}_{\geq l}\right)$ is a one-sided Sturmian sequence.

(iv) The difference sequence $\mathbf{d}(\mathbf{w})$ is a two-sided Sturmian sequence. 
(v) For all $l \in \mathbb{Z}, \mathbf{w}_{>l}$ is a complementation-symmetric one-sided sequence with $p_{\mathbf{w} \geq l}(n)=2 n$.

(vi) The sequence $\mathbf{w}$ is a non-eventually periodic complementation-symmetric two-sided sequence with $p_{\mathbf{w}}(n)=2 n$.

Proof. (ii) $\Leftrightarrow($ i): Clear.

(i) $\Leftrightarrow($ iii $) \Leftrightarrow(v)$ : See Proposition 4.4 .

(iii) $\Leftrightarrow($ iv $): ~(\Rightarrow)$ By Lemma $4.5(2), \mathbf{d}(\mathbf{w})$ is non-eventually periodic. By Lemma $4.5(3), p_{\mathbf{d}(\mathbf{w})}(n)=n+1$. $(\Leftarrow)$ See Proposition 2.1 .

(iv) $\Rightarrow($ ii): Similar with the proof of Proposition $4.4(2) \Rightarrow(3)$ in Chapter 4 of $[8]$.

$(\mathrm{v}) \Rightarrow(\mathrm{vi})$ : Since $\mathbf{w}_{\geq l}$ has unbounded subword complexity, $\mathbf{w}_{>l}$ is noneventually periodic for each $l \in \mathbb{Z}$ by Coven-Hedlund Theorem. By Lemma 4.5 $(2), \mathbf{w}$ is non-eventually periodic. Since $p_{\mathbf{w}_{\geq l}}(n)=2 n$ for all $l, F(\mathbf{w})=F\left(\mathbf{w}_{>l}\right)$ for any $l \in \mathbb{Z}$ and $p_{\mathbf{w}}(n)=2 n$ by Lemma $4.5(3)$. Since complementationsymmetricity depends only on the set of the subwords, w is complementationsymmetric.

$(\mathrm{vi}) \Rightarrow(\mathrm{iv})$ : Let $\mathbf{w}$ be a sequence which is in (vi). Let $\mathbf{s} \in F_{n}(\mathbf{d}(\mathbf{w}))$. There is $\mathbf{u} \in F_{n+1}(\mathbf{w})$ such that $\mathbf{d}(\mathbf{u})=\mathbf{s}$. Since $\mathbf{w}$ is complementation-symmetric, $\widehat{\mathbf{u}} \in F_{n+1}(\mathbf{w})$. By Lemma $4.3, \mathbf{d}(\mathbf{t})=\mathbf{s}$ if and only if $\mathbf{t}=\mathbf{u}$ or $\mathbf{t}=\widehat{\mathbf{u}}$. Therefore, $p_{\mathbf{d}(\mathbf{w})}(n)=\left|F_{n+1}(\mathbf{w})\right| / 2=n+1$. Since $\mathbf{w}$ is non-eventually periodic, $\mathbf{d}(\mathbf{w})$ is non-eventually periodic. Thus, $\mathbf{d}(\mathbf{w})$ is Sturmian.

A two-sided sequence $\mathbf{w}=\cdots w_{-1} w_{0} w_{1} \cdots$ induces a coloring of a 2-regular tree $X$ as follows. Let $V T$ be the set of vertices indexed by $\left\{v_{n}\right\}_{n \in \mathbb{Z}}$. Then the map w $: V T \rightarrow \mathcal{A}$ defined by $v_{n} \mapsto w_{n}$ is a natural coloring of $X$. We will call $b_{\mathbf{w}}$ the subball complexity of $\mathbf{w}$. For an odd length subword $\mathbf{u}$ of $\mathbf{w}$, we will denote by $[\mathbf{u}]$ a colored ball which is induced by $\mathbf{u}$. Note that if $[\mathbf{u}]=\left[\mathbf{u}^{\prime}\right]$, then $\mathbf{u}=\mathbf{u}^{\prime}$ or $\mathbf{u}=\overline{\mathbf{u}^{\prime}}$.

Lemma 4.8. Let $\mathbf{x}=x_{1} \cdots x_{n}$ be an odd length word over $\{0,1\}$. Then, $\overline{\mathbf{d}(\mathbf{x})}=\mathbf{d}(\mathbf{x})$ if and only if $\overline{\mathbf{x}}=\mathbf{x}$.

Proof. First, we will show that $\overline{\mathbf{d}(\mathbf{x})}=\mathbf{d}(\overline{\mathbf{x}})$. Let $\mathbf{d}(\mathbf{x})=d_{1} \cdots d_{n-1}$ and $\mathbf{d}(\overline{\mathbf{x}})=f_{1} \cdots f_{n-1}$. Then, $f_{i}=x_{n-i}-x_{n-i+1}(\bmod 2)=x_{n-i+1}-x_{n-i}(\bmod$ $2)=d_{n-i}$ for all $i=1, \ldots, n-1$, i.e., $d_{n-1} \cdots d_{1} d_{1}=f_{1} \cdots f_{n-1}$. Thus, it is sufficient to show that $\mathbf{d}(\overline{\mathbf{x}})=\mathbf{d}(\mathbf{x})$ if and only if $\overline{\mathbf{x}}=\mathbf{x}$.

By Lemma 4.3, it is enough to show that $\widehat{\mathbf{x}} \neq \overline{\mathbf{x}}$. Let $\overline{\mathbf{x}}=\bar{x}_{1} \bar{x}_{2} \cdots \bar{x}_{n}$. Since $x_{(n+1) / 2}=\bar{x}_{(n+1) / 2}, \widehat{x_{(n+1) / 2}} \neq \bar{x}_{(n+1) / 2}$. Thus, $\widehat{\mathbf{x}} \neq \overline{\mathbf{x}}$.

Droubay-Pirillo Theorem [4] says that a one-sided sequence $\mathbf{v}$ is Sturmian if and only if $F(\mathbf{v})$ contains exactly one palindrome word of even length and two palindrome words of odd length for each positive integer. Note that DroubayPirillo Theorem holds for two-sided Sturmian sequences by Proposition 2.1 and Lemma 4.5(3). 
Theorem 4.9. Let $\mathbf{w}$ be a non-eventually periodic complementation-symmetric two-sided sequence with $p_{\mathbf{w}}(n)=2 n$. Then, $b_{\mathbf{w}}(n)=2 n+2$.

Proof. Let $\mathbf{w}=\cdots w_{-1} w_{0} w_{1} \cdots$ and $\mathbf{d}(\mathbf{w})=\cdots d_{-1} d_{0} d_{1} \cdots$. Then, $p_{\mathbf{w}}(2 n+1)$ $=4 n+2$ and $p_{\mathbf{d}(\mathbf{w})}(2 n)=2 n+1$. By Droubay-Pirillo Theorem, there is a unique palindrome $(2 n)$-word of $\mathbf{d}(\mathbf{w})$ and $2 n$ non-palindrome $(2 n)$-words of $\mathbf{d}(\mathbf{w})$. Two $(2 n+1)$-words of $\mathbf{w}$ correspond to one $(2 n)$-word of $\mathbf{d}(\mathbf{w})$ (see proof of $(\mathrm{vi}) \Rightarrow(\mathrm{iv})$ of Proposition 4.7). By Lemma 4.8, there are exactly two palindrome $(2 n+1)$-words of $\mathbf{w}$ and $4 n$ non-palindrome $(2 n+1)$-words of $\mathbf{w}$.

Let $\mathbf{x}=x_{0} \cdots x_{2 n}$ be a $(2 n+1)$-subword of $\mathbf{w}$ and $\mathbf{d}(\mathbf{x})=d_{0} \cdots d_{2 n-1}$. By Proposition 4.7, $\mathbf{d}(\mathbf{w})$ is Sturmian. Since a one-sided Sturmian sequence is reversible (see Proposition 2.1 .19 on $[7$, p. 54] for details), $\overline{\mathbf{d}(\mathbf{x})} \in F(\mathbf{d}(\mathbf{w}))$. Since $\mathbf{d}(\overline{\mathbf{x}})=\overline{\mathbf{d}(\mathbf{x})}$, there is $\mathbf{u} \in F_{2 n+1}(\mathbf{w})$ such that $\mathbf{d}(\mathbf{u})=\mathbf{d}(\overline{\mathbf{x}})$. By Lemma 4.3, $\mathbf{u}=\overline{\mathbf{x}}$ or $\widehat{\mathbf{u}}=\overline{\mathbf{x}}$. Since $\mathbf{w}$ is complementation-symmetric, $\overline{\mathbf{x}} \in F_{2 n+1}(\mathbf{w})$.

Thus, each non-palindrome $(2 n+1)$-word $\mathbf{x}$ can be paired with its reversed word $\overline{\mathbf{x}}$. Then, there are $2 n$ pairs of $(\mathbf{x}, \overline{\mathbf{x}})$ for non-palindrome words $\mathbf{x}$. Thus, $b_{\mathbf{w}}(n)=(4 n) / 2+2=2 n+2$.

By Theorem 4.9 and Theorem 3.6, there are colorings of a $k$-regular tree with subball complexity $2 n+2$ for each integer $k \geq 2$, which proves Theorem 2 .

\section{Exponential growth}

In this section, we will show that an index map may not preserve linearity of subball complexity. Indeed, we will construct an index map on some sequences whose induced coloring has exponential subball complexity without restriction on the subword complexity of the original sequence, including some sequences of linear subword complexity (see Example 5.2).

Theorem 5.1. Let $k \geq 3$ and let $X$ be a 2-regular tree with a loop attached to each vertex. If $\phi_{0}$ is a non-periodic coloring of $X$ whose 1-balls colored by $[a a a]$ occur only finitely many times for any $a \in \mathcal{A}$, then there exists an index map $i$ on $X$ such that

$$
b_{\phi}(n) \geq(k-1)^{n},
$$

where $\phi$ is the lifting of $\phi_{0}$ to the universal covering of $(X, i)$.

Proof. Let $V X=\left\{v_{s}\right\}_{s \in \mathbb{Z}}$ and $E X=\left\{\left[v_{s}, v_{s \pm 1}\right]: s \in \mathbb{Z}\right\} \cup\left\{\left[v_{s}, v_{s}\right]: s \in \mathbb{Z}\right\}$. Denote a lifting of $v_{s} \in V X$ by $\widetilde{v_{s}}$. There is a ball $K$ in $X$ containing every 1-ball whose coloring occurs only finitely many times.

Throughout the proof, we denote $i^{1}=(p, q, r)$ if $i^{1}$ is an index map on $B_{1}\left(v_{s}\right)-S_{1}\left(v_{s}\right)$ such that $i^{1}\left[v_{s}, v_{s-1}\right]=p, i^{1}\left[v_{s}, v_{s}\right]=q$ and $i^{1}\left[v_{s}, v_{s+1}\right]=r$, (see Figure 2).

We first claim that for any colored 1-ball $\left[B_{1}\left(v_{s}\right)\right]$ appearing infinitely many times, one can construct index maps $i_{t}^{1}, t=1, \ldots, k-1$ which induce $(k-1)$ non-equivalent colored 1-balls $\left[B_{1}\left(\widetilde{v_{s}}\right)\right]$. 
Indeed, since $\left[B_{1}\left(v_{s}\right)\right] \neq[a a a]$ for any $a \in \mathcal{A}$, one of $\phi\left(v_{s-1}\right), \phi\left(v_{s}\right), \phi\left(v_{s+1}\right)$ is different from the others. If $\phi\left(v_{s-1}\right) \neq \phi\left(v_{s+1}\right)$, define $i_{t}^{1}:=(t, 0, k-t)$ on $B_{1}\left(v_{s}\right)-S_{1}\left(v_{s}\right)$ for $1 \leq t \leq(k-1)$. A coloring on $B_{1}\left(\widetilde{v_{s}}\right)$ obtained by $i_{t}^{1}$ has exactly $t$ neighboring vertices of $\widetilde{v_{s}}$ colored by $\phi\left(v_{s-1}\right)$. Thus distinct $i_{t}^{1}$ 's induce (k-1) non-equivalent colorings on $B_{1}\left(\widetilde{v_{s}}\right)$.

If $\phi\left(v_{s-1}\right)=\phi\left(v_{s+1}\right) \neq \phi\left(v_{s}\right)$, define $i_{t}^{1}:=(1, t-1, k-t)$ on $B_{1}\left(v_{s}\right)-S_{1}\left(v_{s}\right)$ for $1 \leq t \leq(k-1)$. As above, there are $(k-1)$ index maps $i_{t}^{1}$ which result in $(k-1)$ distinct colorings on $B_{1}\left(\widetilde{v_{s}}\right)$, which proves the claim (see Figure 3$)$.

To prove the theorem, it is enough to prove the following claim.

Claim: For each $n$, there is a colored $n$-ball $\left[B_{n}\right]$ of $X$ such that

i) $\left[B_{n}\right]$ occurs infinitely many times, say $\left\{B_{n}\left(v_{m_{j}}\right)\right\}_{j=1}^{\infty}$,

ii) there are distinct index maps $i_{j}^{n}: B_{n}\left(v_{m_{j}}\right)-S_{n}\left(v_{m_{j}}\right) \rightarrow \mathbb{N}$, called the index maps on $\left[B_{n}\right]$, such that $\left[B_{n}\left(\widetilde{v_{m_{j}}}\right)\right], i=1, \ldots,(k-1)^{n}$ are all distinct and

iii) there is a ball $K_{n}$ which contains $K_{n-1}$ and $(k-1)^{n} n$-balls equivalent to $\left[B_{n}\right]$ which are outside $K_{n-1}$.

We will prove this claim by using induction on $n$. First choose a colored 1-ball which occurs infinitely many times, say [ $\left.B_{1}\right]$. Define $K_{1}$ in $X$ as a ball containing $K$ and $(k-1)$ 1-balls colored by $\left[B_{1}\right]$ outside $K$. By the claim we proved in the beginning of the proof, we can choose $k-1$ index maps $i_{t}^{1}$, $t=1, \ldots, k-1$ whose induced colored balls are all distinct.

Assume that there is a colored $n$-ball $\left[B_{n}\right]$ and $K_{n}$ as in Claim. Note that there are infinitely many $\left[B_{n}\right]$ outside $K_{n}$. Since there are only finitely many

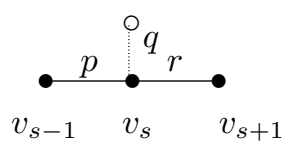

FiguRe 2
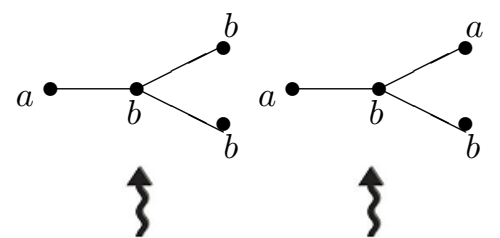

or
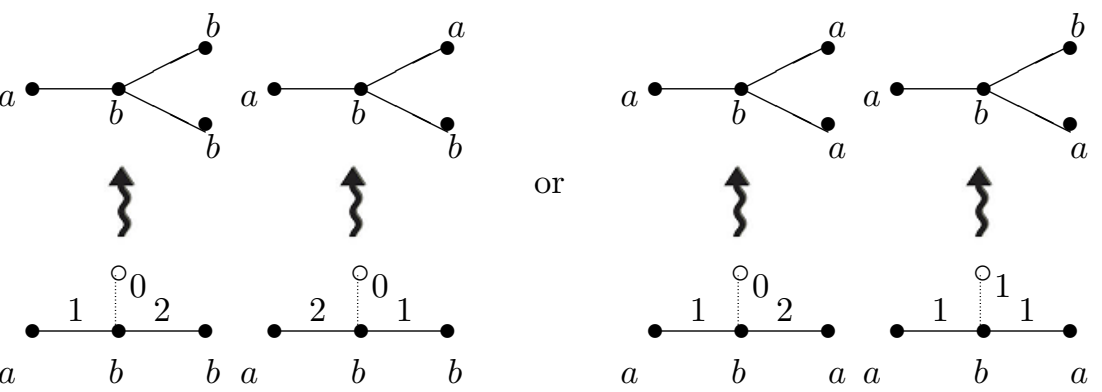

Figure $3 . k=3$ and $n=1$ 

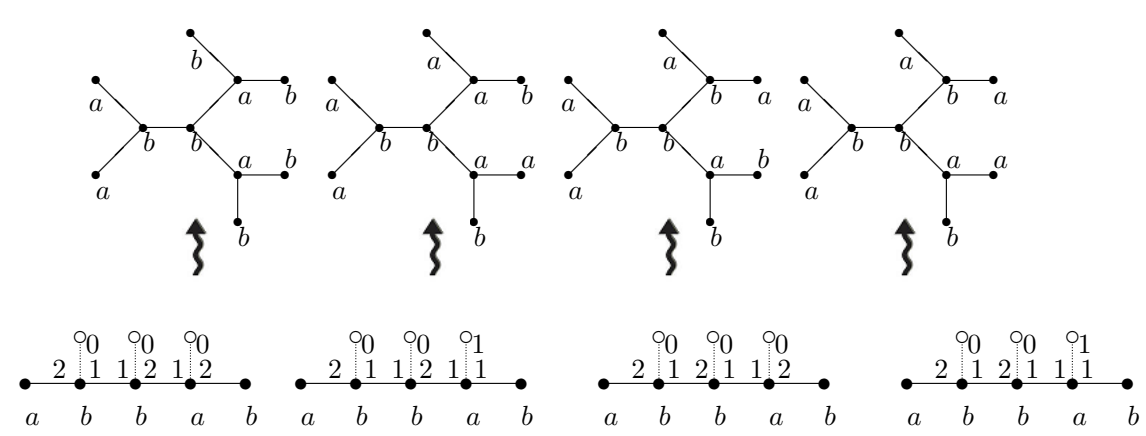

Figure $4 . k=3$ and $n=2$

ways to extend the coloring to $(n+1)$-balls, there is a colored $(n+1)$-ball $\left[B_{n+1}\right]$ outside $K_{n}$ whose central $n$-ball is colored as $\left[B_{n}\right]$ and which occurs infinitely many times.

Define index maps $i_{j, t}^{n+1}:=\left((k-1,0,1), i_{j}^{n}, i_{t}^{1}\right)$ for $j=1, \ldots,(k-1)^{n}$ and $t=1, \ldots,(k-1)$. Consider the index map $i_{j, t}^{n+1}$ on $B_{n+1}\left(v_{s}\right)-S_{n+1}\left(v_{s}\right)$ and $i_{j^{\prime}, t^{\prime}}^{n+1}$ on $B_{n+1}\left(v_{s^{\prime}}\right)-S_{n+1}\left(v_{s^{\prime}}\right)$ where $B_{n+1}\left(v_{s}\right)$ and $B_{n+1}\left(v_{s^{\prime}}\right)$ are colored by $\left[B_{n+1}\right]$. Remark that the definition of $i_{t}^{1}$ depends on the coloring of the 1-ball, and that the map $i_{t}^{1}$ in the definition of $i_{j, t}^{n+1}$ is defined according to the coloring of the rightmost 1-ball in $B_{n+1}\left(v_{s}\right)$.

If $j \neq j^{\prime}$, then $\left[B_{n}\left(\widetilde{v_{s}}\right)\right] \neq\left[B_{n}\left(\widetilde{v_{s^{\prime}}}\right)\right]$, thus $\left[B_{n+1}\left(\widetilde{v_{s}}\right)\right] \neq\left[B_{n+1}\left(\widetilde{v_{s^{\prime}}}\right)\right]$. Suppose that $j=j^{\prime}$ and $t \neq t^{\prime}$. By Lemma 3.5(2), for $l=0,1, \ldots, n-1$,

$$
\begin{aligned}
& {\left[B r_{n+1-l}\left(\widetilde{v_{s+l}}, \widetilde{v_{s+l}}\right)\right]=\left[B r_{n+1-l}\left(\widetilde{v_{s^{\prime}+l}}, \widetilde{v_{s^{\prime}+l}}\right)\right] \text { and }} \\
& {\left[B r_{n+1-l}\left(\widetilde{v_{s+l}}, \widetilde{v_{s+l-1}}\right)\right]=\left[B r_{n+1-l}\left(\widetilde{v_{s^{\prime}+l}}, \widetilde{v_{s^{\prime}+l-1}}\right)\right] .}
\end{aligned}
$$

By (5.1) and (5.2) for $l=n-1$, since $\left[B r_{2}\left(\widetilde{v_{s+n-1}}, \widetilde{v_{s+n}}\right)\right]=\left[B_{1}\left(\widetilde{v_{s+n}}\right)\right] \neq$ $\left[B_{1}\left(\widetilde{v_{s^{\prime}+n}}\right)\right]=\left[B r_{2}\left(\widehat{v_{s^{\prime}+n-1}} \widehat{v_{s^{\prime}+n}}\right)\right],\left[B_{2}\left(\widehat{v_{s+n}}\right)\right] \neq\left[B_{2}\left(\widehat{v_{s^{\prime}+n-1}}\right)\right]$. Since $\left[B r_{2}\left(\widetilde{v_{s+n-1}}, \widetilde{v_{s+n-2}}\right)\right]=\left[B r_{2}\left(\widetilde{v_{s^{\prime}+n-1}}, \widehat{v_{s^{\prime}+n-2}}\right)\right]$ (by $(5.2)$ for $\left.l=n-1\right)$, $\left[B r_{3}\left(v_{s+n-2}, v_{s+n-1}\right)\right] \neq\left[B r_{3}\left(\widehat{v_{s^{\prime}+n-2} v_{s^{\prime}}+n-1}\right)\right]$ (see Lemma 3.4).

Similarly, if $\left[B r_{n+1-l}\left(\widetilde{v_{s+l}}, \widetilde{v_{s+l+1}}\right)\right] \neq\left[B r_{n+1-l}\left(\widetilde{v_{s^{\prime}+l}}, \widetilde{v_{s^{\prime}+l+1}}\right)\right]$, then $\left[B_{n+1-l}\left(\widetilde{v_{s+l}}\right)\right] \neq\left[B_{n+1-l}\left(\widetilde{v_{s^{\prime}+l}}\right)\right]$, thus $\left[B r_{n+2-l}\left(\widetilde{v_{s+l-1}}, \widetilde{v_{s+l}}\right)\right] \neq$ $\left[B r_{n+2-l}\left(\widetilde{v_{s^{\prime}+l-1}}, \widetilde{v_{s^{\prime}+l}}\right)\right]$. By repeating this process, we obtain $\left[B r_{n+1}\left(\widetilde{v_{s}}, \widetilde{v_{s+1}}\right)\right] \neq\left[B r_{n+1}\left(\widetilde{v_{s^{\prime}}}, \widetilde{v_{s^{\prime}+1}}\right)\right] . \quad$ Since $\left[B_{n+1}\left(\widetilde{v_{s}}, \widetilde{v_{s-1}}\right)\right]=$ $\left[B_{n+1}\left(\widetilde{v_{s^{\prime}}}, \widetilde{v_{s^{\prime}}-1}\right)\right]$ and $\left[B_{n+1}\left(\widetilde{v_{s}}, \widetilde{v_{s}}\right)\right]=\left[B_{n+1}\left(\widetilde{v_{s^{\prime}}}, \widetilde{v_{s^{\prime}}}\right)\right],\left[B_{n+1}\left(\widetilde{v_{s}}\right)\right] \neq$ $\left[B_{n+1}\left(\widetilde{v_{s^{\prime}}}\right)\right]$ (see Lemma 3.4).

Therefore, there are $(k-1)^{n+1}$ index maps on $(n+1)$-balls colored by [ $\left.B_{n+1}\right]$ which induce $(k-1)^{n+1}$ distinct colored $(n+1)$-balls in $T$. There is a ball $K_{n+1}$ as in Claim since $\left[B_{n+1}\right]$ occurs infinitely many times. 
Define $i$ on $(k-1)^{n} n$-balls in $K_{n}-K_{n-1}$, which are colored by [ $\left.B_{n}\right]$, by $(k-1)^{n}$ distinct index maps. On the edges which are not yet defined, we can define any indices. By the definition of $i, b_{\phi}(n) \geq(k-1)^{n}$.

Example 5.2. The sequences constructed as in Proposition 4.6 with $\phi=1 / 2$ and $1 / 4<\theta<1 / 2$ satisfies the assumption of Theorem 5.1 (see the proof of Theorem 2 of $[8$, p. 8]).

For example, let $\mathbf{w}$ be the sequence constructed as in Proposition 4.6 with $c=0, \phi=1 / 2$ and $\theta=\sqrt{2} / 4$. Thus $\mathbf{w}=\cdots 101101101001001 \cdots$. Consider $\mathbf{w}$ as a 2-regular tree. By Theorem $4.9, b_{\mathbf{w}}(1)=4$ thus only [001], [011], [101] and [010] are all 1-balls of $\mathbf{w}$. Thus $\mathbf{w}$ satisfies the assumption of Theorem 5.1.

\section{References}

[1] H. Bass, L. Carbone, A. Lubotzky, G. Rosenberg, and J. Tits, Tree Lattices, Birkhäuser, 2001.

[2] J. Cassaigne, Constructing infinite words of intermediate complexity, Developments in language theory, 173-184, Lecture Notes in Comput. Sci., 2450, Springer, Berlin, 2003.

[3] E. Coven and G. Hedlund, Sequences with Minimal Block Growth, Math. Systems Theory 7 (1973), 138-153.

[4] X. Droubay and G. Pirillo, Palindromes and Sturmian words, Theoret. Comput. Sci. 223 (1999), no. 1-2, 73-85.

[5] D. Kim and S. Lim, Subword complexity and Sturmian colorings of regular trees, Ergodic Theory Dynam. Systems 35 (2015), no. 2, 461-481.

[6] H. Kim and S. Park, Toeplitz sequences of intermediate complexity, J. Korean Math. Soc. 48 (2011), no. 2, 383-395.

[7] M. Lothaire, Algebraic Combinatorics on Words, Cambridge University Press, 2002.

[8] G. Rote, Sequences with Subword complexity 2n, J. Number Theory 46 (1994), no. 2, 196-213.

[9] J. Serre, Trees, Springer, 1980.

Seul Bee Lee

Department of Mathematical Sciences

Seoul National University

Seoul 151-747, Korea

E-mail address: sunggar@snu.ac.kr

SeOnheE Lim

Department of Mathematical Sciences

Seoul National University

SeOUl 151-747, Korea

E-mail address: slim@snu.ac.kr 